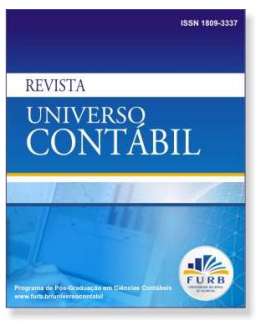

Revista Universo Contábil, ISSN 1809-3337

FURB, v. 6, n.1, p. 142-155, jan./mar., 2010

doi:10.4270/ruc.2010108

Disponível em www.furb.br/universocontabil

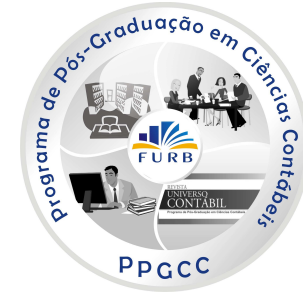

\title{
O NOVO SISTEMA DE AVALIAÇÃO E ACREDITAÇÃO DO ENSINO SUPERIOR: IMPACTO, REPERCUSSÕES E BENEFÍCIOS*
}

\section{THE NEW HIGHER EDUCATION ASSESSMENT AND ACCREDITATION SYSTEM: IMPACT, EFFECTS AND BENEFITS}

\author{
Maria da Conceição da Costa Marques \\ Doutora em Gestão, Especialidade em Contabilidade, pela Universidade Aberta de Coimbra \\ Professora do Instituto Superior de Contabilidade e Administração de Coimbra \\ Departamento de Contabilidade e Gestão \\ Endereço: Quinta Agrícola - Bencanta \\ CEP: 3040-316 - Coimbra - Portugal \\ E-mail: geral@iscac.pt \\ Telefone: +351239802187
}

\section{RESUMO}

A criação de um sistema de garantia de qualidade susceptível de reconhecimento internacional encontra-se entre os desígnios da recente aprovação do regime jurídico da avaliação do ensino superior. Com este regime, o Governo de Portugal pretende instituir parâmetros de avaliação da qualidade, que incluem o ensino ministrado, a qualificação do corpo docente, a estratégia adoptada para garantir a qualidade do ensino, a actividade científica, tecnológica e artística, adequada à missão da instituição e a cooperação internacional. A aprovação destas medidas visa atingir a melhoria da qualidade das instituições de ensino superior, proporcionar informação fundamentada à sociedade sobre o desempenho das instituições, bem como o desenvolvimento de uma cultura institucional interna de garantia de qualidade. Esta avaliação assume carácter obrigatório e realiza-se no quadro do sistema europeu de garantia da qualidade no ensino superior. Os agentes de avaliação são a auto-avaliação, que é realizada por cada estabelecimento de ensino superior e a avaliação externa, que serve de base aos processos de acreditação, a qual é realizada pela agência de avaliação e acreditação para a garantia da qualidade do ensino superior. A Agência

Artigo recebido em 17.05.2009. Revisado por pares em 28.07.2009. Reformulado em 15.09.2009. Recomendado em 23.09.2009 por Ilse Maria Beuren (Editora). Publicado em 02.02.2010. Organização responsável pelo periódico: FURB. 
de Avaliação e Acreditação do Ensino Superior, foi criada legalmente em novembro de 2007. A acreditação de instituições de ensino superior e dos seus ciclos de estudos, é obrigatória e pode ser da iniciativa da Agência ou partir das próprias instituições de ensino superior, que se mostrem interessadas. Neste artigo propomo-nos apresentar e desenvolver os recentes desafios que se colocam às instituições de ensino superior, dando especial enfoque à fase em que a implementação destas medidas governamentais se encontre.

Palavras-chave: Acreditação. Avaliação. Ensino superior.

\section{ABSTRACT}

The creation of a quality assurance system capable of international recognition is among the designs of the recent approval of the higher education legal system of evaluation. With this scheme, the Government intends to establish parameters for assessing quality, which include education, the qualifications of the faculty, the strategy adopted to ensure the quality of education, the scientific, technological and artistic activity, appropriated to the mission of the institution and international cooperation. The adoption of these measures aimed at achieving improved quality of higher education institutions, to provide information to the company founded on the performance of institutions, as well as the development of an internal institutional culture of quality assurance. This evaluation is mandatory and takes place in the European system of quality assurance in higher education. The agent of such assessment are the self-assessment, which is performed by each institution of higher education, and the external assessment, serving as basis for the process of accreditation, which is performed by the assessment and accreditation agency to assure the quality in higher education. The Agency for Assessment and Accreditation of Higher Education was legally established in November 2007. The accreditation of higher education institutions and their courses is mandatory and can be an initiative of the Agency or of the higher education institutions themselves, which show interest. It is proposed in this paper to present develop the recent challenges faced by higher education institutions, giving special focus to the stage where the implementation of those government's measures are.

Keywords: Accreditation. Assessment. Higher Education.

\section{INTRODUÇÃO}

A criação de um sistema de garantia de qualidade susceptível de reconhecimento internacional encontra-se entre os desígnios da recente aprovação do regime jurídico da avaliação do ensino superior. Com este regime, o Governo de Portugal pretende instituir parâmetros de avaliação da qualidade, que incluem o ensino ministrado, a qualificação do corpo docente, a estratégia adoptada para garantir a qualidade do ensino, a actividade científica, tecnológica e artística, adequada à missão da instituição e a cooperação internacional.

A implementação de um sistema nacional de avaliação e acreditação do ensino superior, segundo Santos (2007), foi tratada em grande profundidade em 2004, tanto a nível de conceptualização e de definição de princípios orientadores como a nível de operacionalização, num trabalho que foi articulado de perto com o Ministério da Ciência e do Ensino Superior.

A aprovação destas medidas visa atingir a melhoria da qualidade das instituições de ensino superior, proporcionar informação fundamentada à sociedade sobre o desempenho das 
instituições, bem como o desenvolvimento de uma cultura institucional interna de garantia de qualidade. Esta avaliação assume carácter obrigatório e realiza-se no quadro do sistema europeu de garantia da qualidade no ensino superior.

Os agentes de avaliação são a auto-avaliação, que é realizada por cada estabelecimento de ensino superior, e a avaliação externa, que serve de base aos processos de acreditação, a qual é realizada pela agência de avaliação e acreditação para a garantia da qualidade do ensino superior.

Com a introdução da figura da Agência de Acreditação pelo Decreto-Lei no 74/2006, o assunto voltou a ser alvo de discussão, tendo ganho especial força após a publicação do Relatório da Quality Assurance of Higher Education in Portugal, produzido no European Network for Quality Assurance (ENQA), através do qual se recomendou largamente a sua concretização.

A Agência de Avaliação e Acreditação do Ensino Superior, foi criada legalmente em novembro de 2007. A acreditação de instituições de ensino superior e dos seus ciclos de estudos é obrigatória e pode ser da iniciativa da Agência ou partir das próprias instituições de ensino superior, que se mostrem interessadas.

Neste estudo, além de se fazer uma revisão da literatura acerca dos conceitos de qualidade, avaliação e acreditação, damos especial ênfase ao novo paradigma de garantia da qualidade do ensino superior em Portugal, cujo suporte legislativo foi aprovado no ano de 2007.

Sobre o processo de avaliação e acreditação das instituições de ensino superior, recaem diferentes expectativas, que apenas poderão ser satisfeitas com a implementação do processo.

\section{GARANTIA DA QUALIDADE}

Actualmente, já não se discute a necessidade de avaliar as diferentes actividades das Instituições de Ensino Superior (IES). Os programas de avaliação são amplamente aceites; o que se debate são os aspectos concretos em que se materializa tal avaliação.

Não existe um modelo único de avaliação que possa ser aplicado em qualquer país. Há que considerar a sua realidade social, cultural e política, bem como os aspectos que favorecem ou dificultam a sua implementação (NAUTA et al., 2004).

Um aspecto importante a considerar é a influência que o resultado da avaliação possa vir a ter no financiamento das IES. De acordo com Bricall (2000, p. 361), a conjugação da autonomia institucional com a responsabilidade social e do papel que têm na avaliação da qualidade, constitui o meio mais adequado para equilibrar e harmonizar esse binómio.

Uma vez que não é previsível o crescimento quantitativo das IES, o grande desafio que se lhes coloca consiste na garantia da qualidade dos serviços que oferece. Assim, as Administrações Públicas e as instituições de ensino superior, em colaboração com outros sectores económicos e sociais, devem envidar esforços e sensibilizar a comunidade universitária sobre uma cultura de qualidade, como elemento de equilíbrio entre autonomia e responsabilidade social.

Bricall (2000, p. 363) argumenta que ao conceito de qualidade podem atribuir-se diferentes acepções. No âmbito universitário considera-se qualidade algumas características dos professores, estudantes, recursos financeiros, instalações, propinas, a sua história, ou apenas a sua fama e reconhecimento público.

A qualidade pode significar conformidade com os padrões pré-estabelecidos, ou adequação a um dado objectivo ou finalidade (fitness for purpose). A qualidade pode estar relacionada com a eficiência e os custos suportados pela Instituição. 
A qualidade é definida como a aptidão para satisfazer os utilizadores, destinatários ou clientes; esta é uma definição plenamente aceita na indústria e em serviços, mas com alguma resistência de aceitação no âmbito universitário.

Surge, por conseguinte, um novo conceito de qualidade, relacionado com a capacidade de transformação e mudança das IES, que coloca a ênfase na necessidade de gestão da mudança perante a procura da sociedade, a melhora e reengenharia de processos, a adaptação às novas tecnologias de informação e comunicação, a adaptação dos recursos humanos e de novas formas de organização institucional (WESTERHEIJDEN; STENSAKER, ROSA, 2007a). Os sistemas implementados devem ter como escopo fundamental a melhoria da qualidade que deve incorporar as normas reguladoras ou de acreditação.

O surgimento de novos conceitos de qualidade não exclui a validade dos conceitos anteriores. Daí que o conceito de qualidade seja um conceito multidimensional e complexo.

Nas IES, os sistemas de qualidade devem cumprir determinadas funções (WESTERHEIJDEN; STENSAKER, ROSA, 2007b), a saber:

a) preparar os mecanismos para a acreditação de instituições e programas;

b) melhorar a docência, a investigação e a administração;

c) servir de instrumento de prestação de contas ao Governo e à sociedade;

d) fornecer informação pública e transparência aos estudantes e ao mercado laboral;

e) operar como mecanismo de diferenciação no financiamento das IES por parte do Estado.

Os métodos a utilizar podem variar. Assim, pode optar-se por um modelo de autoavaliação, em que a responsabilidade na iniciativa e a execução recaem na IES, por um modelo de controlo externo a exercer pelo Governo ou ainda um modelo misto, em uso nalguns países, que se baseia na criação de agências independentes criadas pelo Governo. A tendência é a combinação duma primeira autoavaliação interna com a avaliação externa (PROITZ; STENSAKER; HARVEY, 2004).

É desejável que o sistema de qualidade combine o uso simultâneo da autoavaliação interna e a avaliação externa, incluindo a apresentação pública do relatório. A avaliação externa deve ser efectuada por entidades autónomas, independentes da Administração do Estado e das IES (STENSAKER, 2003).

\section{EXPERIÊNCIAS DE AVALIAÇÃO}

\subsection{Internacionais}

A avaliação das instituições de ensino superior constitui uma prática generalizada nos países europeus e tem larga tradição nos países anglo-saxónicos.

Nos Estados Unidos, foram as próprias instituições universitárias que iniciaram há bastantes anos investigações sobre a eficiência do seu próprio funcionamento, a revisão de programas e o planeamento estratégico. Procedia-se à acreditação por agências regionais especializadas, muitas vezes de carácter privado, com base na revisão da estrutura organizacional e em visitas de grupos avaliadores externos.

Segundo Bricall (2000, p. 367), mais de dois terços dos Estados dispõem de um sistema de avaliação da qualidade para instituições de ensino superior no seu território. Um aspecto importante do processo de avaliação, é a valorização dos resultados do processo educativo pelos estudantes, que representa para estes um valor acrescentado significativo.

$\mathrm{Na}$ Austrália, o modelo de avaliação é distinto. Existe uma agência estatal, a Higher Education Research and Development Society (HERDSA), que segue as recomendações da Commonwealth Tertiary Education Comission (CTEC), organizações responsável pelo ensino 
superior, que prepara a metodologia de revisão a realizar pelas universidades, de molde a que se realizem autoavaliações de três em três anos.

O sistema de avaliação no Reino Unido, teve início por volta dos anos 90, com a criação da Academic Audit Unit pela Conferência britânica de Reitores (Committee of ViceChancellors and Principals - CVCP), que veio a integrar em 1992 o Higher Education Quality Council (HEQC). O sistema de avaliação auto-regulada no Reino Unido tem por base o uso de indicadores de rendimento (MORAN, 2007)).

Na Dinamarca criou-se em 1992 um centro para garantir a qualidade e avaliação do ensino superior, dependente do Ministro da Educação. Realiza as avaliações dos programas de ensino e define as áreas prioritárias de actuação. Em resultado da avaliação é produzido um relatório de avaliação que inclui recomendações consideradas oportunas. As avaliações são pedidas pelos conselhos de educação e a implementação dos programas educativos decorrem do próprio Ministro da Educação. As avaliações têm carácter periódico.

Na França, o Comité National D'Évaluation, criado em 1984, é um organismo autónomo independente. Encarrega-se de avaliar os aspectos docentes e de investigação das universidades, mas sem competência para avaliar as pessoas, para aprovar os curricula ou para distribuir as subvenções do Estado. Não se exige que as universidades elaborem um relatório prévio à sua visita, devendo apenas disponibilizar os dados necessários à avaliação, sendo depois produzido um relatório público. Este Comité é constituído por membros destacados da comunidade académica e por individualidades doutros organismos do Estado.

$\mathrm{Na}$ Holanda, o sistema de gestão da qualidade teve início na década de 1980, com a publicação dum documento intitulado Ensino Superior: Autonomia e Qualidade, emitido pelo Ministério da Educação, Cultura e Ciência. De acordo com este, todas as instituições de ensino superior devem responsabilizar-se por assegurar a qualidade dos seus programas educativos e de avaliação interna, que deverão ser tornados públicos. A avaliação processa-se em ciclos de seis anos e combina a autoavaliação e a avaliação externa, devendo o relatório final ser tornado público. A Administração Pública deve assumir um controlo remoto sobre o sistema de ensino superior. Em 1987 foi implementado um sistema de revisão da qualidade dos programas educativos, devendo ser objecto de implantação as recomendações de qualidade.

Em Espanha, em finais da década de 1980, o Conselho de Universidades iniciou uma linha de trabalho para desenhar e implementar um programa de avaliação com o objectivo de dotar o sistema universitário de padrões de qualidade, sobretudo ao nível dos recursos humanos e materiais. Não se tendo atingido os objectivos propostos, a partir de 1990, o Conselho optou por seguir os padrões de avaliação baseados em modelos de outros países, de que resultou o Programa Experimental de Avaliação da Qualidade das Universidades, que desenvolveu em 1992 e 1994, e a participação logo a seguir no Projecto Piloto da União Europeia. Este processo teve continuidade institucional no Plano Nacional de Avaliação da Qualidade, aprovado pelo Governo em 1995.

Conforme refere Bricall (2000, p. 369), têm surgido iniciativas por parte de instituições, como é o caso da Universidade Tecnológica de Delft, que concede elevada importância à avaliação da sua qualidade, entendida como cumprimento dos objectivos institucionais, como seja a qualidade da docência e da investigação, eficácia da gestão universitária, a qualidade dos recursos humanos e a organização dos seus sistemas de decisão.

A Universidade de Twent, em conjunto com outras dez universidades europeias, agrupadas no European Consortium of Innovative Universities, iniciaram um programa piloto de acreditação internacional. Defendem um sistema de revisão e reconhecimento internacional entre universidades parecidas e para programas inovadores. Trata-se de um modelo que 
permite às universidades membros da rede actuar como agências externas de acreditação e de reforço mútuo dos processos de avaliação e gestão da qualidade (ORSINGHER, 2006).

Estas experiências reforçam a ideia de que existe uma necessidade crescente de acreditação internacional. Trata-se de um elemento chave no futuro, sobretudo para algumas universidades e cursos, tanto ao nível do seu país como numa projecção internacional. $\mathrm{O}$ sistema de qualidade nas IES deverá facilitar a transparência (SOGUEL; JACCARD, 2007).

\subsection{Em Portugal}

A necessidade de avaliação do sistema de ensino superior começou a ser reconhecida formalmente em 1986, tendo a Assembleia da República nesse mesmo ano aprovado a primeira legislação nesse domínio, que a revisão constitucional de 1997 veio a contemplar.

Nesse período, deu-se início a um debate público sobre a matéria, através do Conselho de Reitores das Universidades Portuguesas (CRUP), tendo as universidades impulsionado um processo de autoavaliação, através de uma experiência piloto, que veio a ser incluída na Lei $\mathrm{n}^{\circ}$ 38/94, de 21 de novembro 1994, Lei da Avaliação do Ensino Superior, tendo sido criada a Fundação das Universidades Portuguesas (FUP).

A Constituição de 1996 contemplou o alargamento da avaliação a todos os subsistemas, tendo sido criado um Grupo de Reflexão e Acompanhamento do Processo de Avaliação das Instituições do Ensino Superior, com a missão de propor as regras e os princípios gerais que assegurassem a concretização do sistema de avaliação, a constituição das entidades representativas e o funcionamento do sistema em termos coesos e credíveis. Cabialhe também a elaboração do guião para a auto-avaliação, de um primeiro documento estratégico e de um projecto de diploma legal que está na origem do Decreto-Lei ${ }^{\circ}$ 205/98, de 11 de julho de 1998, que criou o Conselho Nacional de Avaliação do Ensino Superior (CNAVES).

O processo de avaliação é aplicável a todas as instituições do ensino superior universitário e politécnico, público, concordatário, privado e cooperativo e ao militar. Tem por alcance fomentar a melhoria da qualidade das actividades desenvolvidas, prestar esclarecimentos à comunidade académica e à sociedade em geral. Encontra-se também entre os seus objectivos assegurar um conhecimento mais rigoroso e um diálogo mais transparente entre as instituições de ensino superior, bem como contribuir para o ordenamento da rede.

O processo de avaliação desencadeia-se em duas fases: a auto-avaliação e a avaliação externa, culminando com a avaliação global da instituição. A meta-avaliação poderá ser efectuada por entidade externa nacional ou estrangeira, em coordenação com o CNAVES, na qualidade de órgão nacional de cúpula do sistema, que tem por fim garantir a completa harmonia, coesão e credibilidade de todo o processo de avaliação e acompanhamento do ensino superior, tendo em vista a observância dos padrões de excelência a que deve corresponder o funcionamento global do sistema.

Compete ao CNAVES apreciar a coerência global do sistema de avaliação, a partir de indicadores utilizados nas várias modalidades de ensino, devendo produzir relatórios prospectivos e recomendações de racionalização e melhoria do sistema de ensino superior.

Em resultado dum processo de reformas encetadas pelo Governo, o CNAVES foi extinto pelo Decreto-Lei no 369/2007, de 5 novembro 2007, que criou a Agência de Avaliação e Acreditação do Ensino Superior. Com a aprovação da Lei no 38/2007, de 16 de agosto 2007, institui-se um sistema nacional de garantia da qualidade do ensino superior reconhecido internacionalmente. 


\section{O PROCESSO DE GARANTIA DA QUALIDADE DO ENSINO SUPERIOR}

\subsection{Avaliação versus Acreditação}

Hoje em dia, as dificuldades existentes para distinguir avaliação de acreditação, tendem a esbater-se, deixando até de fazer sentido. Avaliação e acreditação possuem objectivos complementares. A avaliação visa a monitorização e melhoria constante da qualidade, pelo que representa uma função nobre e de grande interesse para a comunidade, como seja a promoção da qualidade do ensino, investigação e aç̧ão cultural, desenvolvidos no seio das instituições, para potenciar os resultados com os recursos disponíveis. Por seu lado, a acreditação pretende garantir o cumprimento dos requisitos mínimos que levam ao reconhecimento oficial do curso ou instituição avaliados (SANTOS, 2007).

Por conseguinte, a avaliação e acreditação são processos indissociáveis. O processo de avaliação transmite publicamente à sociedade uma mensagem sobre a qualidade de uma instituição ou curso. Já a acreditação fornece à sociedade uma informação relevante sobre se determinado curso cumpre os critérios mínimos de qualidade para o seu reconhecimento.

\subsection{O sistema de Avaliação e Acreditação}

Não há um modelo exclusivo de avaliação e acreditação que seja de aplicação geral em todas as circunstâncias e países. Um aspecto a considerar prende-se com os indicadores de rendimento. Estes podem ser de três tipos:

a) indicadores de rendimento interno - em que se considera a taxa de alunos que concluíram a graduação, a captação de fundos para a investigação ou a taxa de sucesso dos graus superiores e o tempo que os alunos levam a completá-los;

b) indicadores de rendimento externo - onde se considera a taxa de empregabilidade dos licenciados, as publicações e trabalhos que os professores apresentam em congressos ou o registo de patentes, invenções ou consultoria;

c) indicadores de rendimento operacional - onde se aprecia o número de alunos por turma, o número de computadores disponíveis para os alunos ou a carga horária dos docentes.

A avaliação deve ser vista sob duas perspectivas: do exterior ao sistema universitário, em que se deve assegurar que a sociedade e em particular os estudantes conhecem a qualidade e a oferta dos programas académicos. No interior da instituição, a avaliação constitui um instrumento para observação dos pontos fortes e fracos e para impulsionar a estratégia a seguir para se atingirem maiores níveis de qualidade (NAUTA et al., 2004).

Os aspectos precedentes, apesar de importantes, não esgotam o processo de avaliação. Esta serve também para que as instituições consigam atingir maiores níveis de financiamento por parte dos governos e outras fontes de financiamento.

Substituir o tradicional processo de homologação e autorização por outro de acreditação, significa transformar progressivamente os mecanismos de intervenção prévia por instrumentos de auditoria, em que se avalia a qualidade da gestão (CARR; HAMILTON; MEADE, 2005). O crescente papel que desempenha a mobilidade dos estudantes e dos profissionais, evidencia a importância dos programas de qualidade e de procedimentos de acreditação que garantam a continuação de estudos ou a prática duma profissão. 


\subsection{O papel da ENQA}

The European Network for Quality Assurance in Higher Education (Rede Europeia para a Garantia da Qualidade no Ensino Superior) foi criada no ano de 2000 para promover a cooperação europeia no domínio da garantia de qualidade. Em novembro de 2004, a Assembleia Geral transformou a Rede na Association for Quality Assurance in Higher Education (ENQA) (Associação Europeia para a Garantia da Qualidade no Ensino Superior). A ideia de criação duma associação europeia teve origem no Projecto Piloto de Avaliação da qualidade do Ensino Superior (1994-95), que demonstrou o valor da partilha de experiências e o desenvolvimento na área da garantia de qualidade. Posteriormente, foi reforçado esse sentido pela Recomendação do Conselho (98/561/CE, de 24 de setembro de 1998) sobre a cooperação europeia na garantia da qualidade do ensino superior e pela Declaração de Bolonha de 1999. A Comissão Europeia, através da concessão de apoios, tem financiado as actividades da ENQA desde o início.

Conforme consta do website da ENQA (http://www.enqa.eu/agencies.lasso), são actualmente membros desta Associação os que constam no Quadro 1.

Em setembro de 2008 encontravam-se como candidatas a full members da ENQA dez instituições. Porém, antes de assumirem o estatuto de membros, estas têm de estar dois anos como candidatas a membros.

A ENQA tem como objectivo fortalecer a cooperação comunitária em matéria de qualidade. É através da sua Assembleia Geral, em que estão representadas as agências de qualidade e instituições nacionais similares de diversos países europeus, que procura atingir tal finalidade, se bem que o seu espectro de actividades seja bem mais amplo.

Depois da Declaração de Bolonha, o papel de ENQA foi aumentando. O facto de a garantia da qualidade constituir um dos pilares da construção do Espaço Europeu de Educação Superior, fez com que a organização se tornasse numa peça importante no processo e, como tal, uma das organizações mais activas. Para reforço deste seu papel, a ENQA realiza vários workshops, prepara relatórios em decurso dos mesmos, participa em grupos relacionados com o processo de Bolonha e propõe ampliar as suas actividades no que respeita à garantia da qualidade em actividades relacionadas com o e-learning.

A ENQA conta com o apoio da Comissão Europeia para o financiamento das suas actividades. Entre estas encontra-se a conclusão de diversos projectos relacionados com a qualidade, bem como o início de outros. Já concluiu avaliações externas aos membros da Rede, bem como um estudo sobre convergência da qualidade na Europa, entre outros. Paralelamente à realização de projectos, encontra-se a elaboração de documentos sobre a qualidade, que se revelaram fundamentais no desenvolvimento do processo de Bolonha.

Em Portugal, a ENQA conduziu um processo de avaliação ao ensino superior, a pedido do Governo, que culminou com um relatório. Segundo Azevedo (2006, p. 44) na apresentação do relatório, o responsável da ENQA pelo projecto referiu o seguinte: Institutional accreditation should be considered in order to steer and regulate the number and quality of Higher Education Institutions. Para que tal se torne viável, é necessário que se opere uma mudança significativa no sistema de ensino superior em geral e das instituições em particular. Algumas mudanças, já foram todavia iniciadas. 


\begin{tabular}{|c|c|}
\hline \multicolumn{2}{|c|}{ Full Members Agencies } \\
\hline Austria & Ireland \\
\hline AAC - Austrian Accreditation Council, Vienna & HEA - Higher Education Authority, Dublin 4 \\
\hline $\begin{array}{l}\text { AQA - Austrian Agency for Quality Assurance, } \\
\text { Vienna }\end{array}$ & $\begin{array}{l}\text { HETAC - Higher Education and Training Awards } \\
\text { Council, Dublin } 2\end{array}$ \\
\hline FHR - Fachhochschulrat, Vienna & $\begin{array}{l}\text { NQAI - National Qualifications Authority of } \\
\text { Ireland, Dublin } 1\end{array}$ \\
\hline Belgium & Italy \\
\hline $\begin{array}{l}\text { VLHORA - Council of Flemish Institutions of Higher } \\
\text { Education, Brussels }\end{array}$ & $\begin{array}{l}\text { CNVSU - Comitato Nazionale per la Valutazione } \\
\text { del Sistema Universitario, Rome }\end{array}$ \\
\hline VLIR - Flemish Interuniversity Council, Brussels & Latvia \\
\hline Cyprus & $\begin{array}{l}\text { HEQEC - Higher Education Quality Evaluation } \\
\text { Centre, Riga }\end{array}$ \\
\hline $\begin{array}{l}\text { CEEA - Council of Educational Evaluation- } \\
\text { Accreditation, Nicosia }\end{array}$ & The Netherlands \\
\hline The Czech Republic & NQA - Netherlands Quality Agency, Utrecht \\
\hline Accreditation Commission Czech Republic, Prague 1 & $\begin{array}{l}\text { NVAO - Accreditation Organisation of the } \\
\text { Netherlands and Flanders, The Hague }\end{array}$ \\
\hline Denmark & $\begin{array}{l}\text { QANU - Quality Assurance Netherlands } \\
\text { Universities, Utrecht }\end{array}$ \\
\hline EVA - Danish Evaluation Institute, Copenhagen & Norway \\
\hline Estonia & $\begin{array}{l}\text { NOKUT - Norwegian Agency for Quality } \\
\text { Assurance in Education, Oslo }\end{array}$ \\
\hline $\begin{array}{l}\text { Estonian Higher Education Accreditation Center, } \\
\text { Tallinn }\end{array}$ & The Slovak Republic \\
\hline Finland & $\begin{array}{l}\text { Accreditation Commission of the Slovak Republic, } \\
\text { Bratislava }\end{array}$ \\
\hline $\begin{array}{l}\text { FINHEEC - Finnish Higher Education Evaluation } \\
\text { Council, Helsinki }\end{array}$ & Spain \\
\hline France & $\begin{array}{l}\text { AGAE - Agency for Quality Assurance in Higher } \\
\text { Education and Research of Andalusia, Córdoba }\end{array}$ \\
\hline $\begin{array}{l}\text { AERES - Evaluation Agency for Research and Higher } \\
\text { Education, Paris }\end{array}$ & $\begin{array}{l}\text { ANECA - National Agency for Quality Assessment } \\
\text { and Accreditation of Spain, Madrid }\end{array}$ \\
\hline $\begin{array}{l}\text { CTI - Commission des Titres d'Ingénieur, Neuilly sur } \\
\text { Seine }\end{array}$ & $\begin{array}{l}\text { AQU - Agency for Quality Assurance in the } \\
\text { Catalan University System, Barcelona }\end{array}$ \\
\hline Germany & Sweden \\
\hline $\begin{array}{l}\text { ACQUIN - Accreditation, Certification and Quality } \\
\text { Assurance Institute, Bayreuth }\end{array}$ & $\begin{array}{l}\text { NAHE - National Agency for Higher Education, } \\
\text { Stockholm }\end{array}$ \\
\hline $\begin{array}{l}\text { ASIIN - Accreditation Agency Specialised in } \\
\text { Accrediting Degree Programmes in Engineering, } \\
\text { Informatics, the Natural Sciences and Mathematics, } \\
\text { Duesseldorf }\end{array}$ & Switzerland \\
\hline $\begin{array}{l}\text { EVALAG - Stiftung Evaluationsagentur Baden- } \\
\text { Wuerttemberg, Mannheim }\end{array}$ & $\begin{array}{l}\text { OAQ - Center for Accreditation and Quality } \\
\text { Assurance of the Swiss Universities, Bern }\end{array}$ \\
\hline $\begin{array}{l}\text { FIBAA - Foundation for International Business } \\
\text { Administration Accreditation, Bonn }\end{array}$ & UK \\
\hline GAC - German Accreditation Council, Bonn & $\begin{array}{l}\text { QAA - Quality Assurance Agency for Higher } \\
\text { Education, Gloucester }\end{array}$ \\
\hline $\begin{array}{l}\text { ZEvA - Central Evaluation and Accreditation Agency } \\
\text { Hannover, Hannover }\end{array}$ & European agencies and organizations \\
\hline Hungary & EUA - European University Association \\
\hline HAC - Hungarian Accreditation Commi & \\
\hline
\end{tabular}

Fonte: ENQA (http://www.enqa.eu/agencies.lasso). 


\section{AVALIAÇÃO E ACREDITAÇÃO EM PORTUGAL: UM NOVO PARADIGMA}

\subsection{O Que Foi Feito: Metodologia e Aspectos Relevantes}

O Relatório elaborado em 2005 pela European Association for Quality Assurance in Higher Education (ENQA) no âmbito da concretização do Processo de Bolonha, intitulado Standards and Guidelines for Quality Assurance in the European Higher Education Area refere-se aos princípios gerais para a garantia de qualidade do ensino superior na Europa.

Em sequência deste documento, foi produzido em novembro de 2006 um relatório sobre a garantia da qualidade do ensino superior em Portugal elaborado pela referida Associação Europeia, a pedido do Governo, que procedeu à avaliação das práticas neste domínio levadas a cabo no âmbito do Conselho Nacional de Avaliação do Ensino Superior e formulou recomendações ao Governo sobre a organização, método e processos de um novo sistema de acreditação, denominado Quality Assurance of Higher Education in Portugal. As principais recomendações do relatório contemplam aspectos como:

a) criação de uma agência nacional forte e independente;

b) publicação dos resultados da avaliação;

c) produção de relatórios resumidos cada dois ou três anos;

d) profissionalismo e consistência do trabalho da agência de avaliação e acreditação;

e) uma agência com um 'staff' altamente qualificado;

f) recurso a peritos internacionais;

g) inclusão dos estudantes no processo de avaliação da qualidade;

h) envolvimento dos 'stakeholders';

i) disponibilidade para cooperar em trabalhos em rede, no âmbito europeu e internacional;

j) procedimentos internos de responsabilidade de acordo com os padrões europeus;

k) pré-acreditação e acreditação baseada em critérios de relevância e interesse nacional;

1) financiamento da nova agência de avaliação da qualidade;

m) focus' nos critérios específicos da avaliação;

n) o processo para a definição dos critérios de acreditação;

o) definição dos critérios de acreditação;

p) níveis de acreditação e seguimento;

q) consequências da acreditação;

r) duração do ciclo de acreditação;

s) possibilidade de recurso das decisões da acreditação.

Em dezembro de 2006, veio a público o Relatório de avaliação do sistema de ensino superior português, elaborado pela Organização para a Cooperação e Desenvolvimento Económico (OCDE), a pedido do Governo, intitulado Reviews of National Policies for Education: Tertiary Education in Portugal.

No que se refere à qualidade e excelência no ensino superior e em ciência e tecnologia, o relatório refere que "o sistema português de ensino superior deve ter como objectivo alcançar níveis superiores de qualidade e excelência, particularmente nos sectores politécnico e privado".

Segundo o mesmo relatório todas as instituições deverão que dispõem de níveis de qualidade em termos de liderança, desenvolvimento curricular, desempenho pedagógico, viabilidade financeira.

No que se prende com a acreditação de novos programas, estes deverão ser objecto de rigorosas avaliações por autoridades independentes e externas, conforme proposto pela 
ENQA. Já as avaliações externas deverão ser efectuadas de forma regular a todos os programas de estudo e a todas as instituições.

No relatório da OCDE é realçada a necessidade de fortalecer a exposição das universidades a ambientes de crescente competitividade e prestação de contas, nomeadamente ao nível do desempenho científico e pedagógico. Por seu lado, as unidades de I\&D deverão ser reforçadas de modo a criar maior e melhor especialização em determinadas áreas científicas. Pretende-se desta forma garantir as necessárias massas críticas ao desenvolvimento científico e à especialização do conhecimento que emerge.

Refira-se, que é pelo Decreto-Lei $n^{\circ}$. 74/2006, de 24 de março 2006, onde se aprova o regime jurídico dos graus e diplomas, que se fixam os princípios gerais da acreditação das instituições de ensino superior e dos seus ciclos de estudos.

\subsection{O Regime Jurídico da Avaliação do Ensino Superior}

O Decreto-Lei $n^{\circ} 38 / 2007$, de 16 de agosto 2007, estabelece o regime jurídico da avaliação do ensino superior. Segundo este diploma, a avaliação da qualidade é obrigatória e realiza -se no quadro do sistema europeu de garantia da qualidade no ensino superior. Incide sobre os estabelecimentos de ensino superior e as suas unidades orgânicas e sobre os ciclos de estudos. A avaliação da qualidade reveste as formas de auto-avaliação e avaliação externa.

A avaliação será levada a cabo pelas próprias instituições e pela Agência de Avaliação e Acreditação do Ensino Superior. Prevê-se a avaliação do ensino ministrado, da qualificação do corpo docente, da cooperação internacional, da actividade científica e tecnológica, entre outros, tal como consta da referida lei.

O sistema de avaliação de qualidade inclui os estudantes e entidades externas, como as ordens e outras associações públicas profissionais. Os resultados da avaliação serão públicos.

Por sua vez, através do Decreto-Lei no 369/2007, de 5 de novembro 2007, é criada a Agência de Avaliação e Acreditação do Ensino Superior, que assumirá a responsabilidade pelos procedimentos de garantia da qualidade desse grau de ensino, designadamente os de avaliação e acreditação, bem como a inserção de Portugal no sistema europeu de garantia da qualidade do ensino superior.

A Agência é uma fundação de direito privado, dotada de regras claras de independência, representatividade e competência técnico-científica, sendo responsável pela avaliação e acreditação das instituições e cursos de ensino superior.

A sua criação constitui um passo importante para a implementação de um sistema nacional de garantia de qualidade no ensino superior, independente, reconhecido internacionalmente e que abarque todas as suas instituições.

A Agência será dirigida por um conselho de administração composto por personalidades de reconhecido mérito e especialização na área da garantia da qualidade do ensino superior e da ciência. Cabe a este conselho as decisões em matéria de avaliação e de acreditação de cursos ou estabelecimentos de ensino, função exercida com total independência.

Ao conselho consultivo caberá a representação dos interesses das entidades avaliadas, sendo composto por membros designados pelos estabelecimentos de ensino superior, pelos estudantes, pelas associações profissionais e por outros parceiros sociais.

Segundo o Ministério da Ciência, Tecnologia e Ensino Superior (MCTES) (www.mctes.pt), o regime jurídico de garantia da qualidade do ensino superior:

a) introduz um verdadeiro sistema de avaliação externa, independente das instituições de ensino, e onde não se confundem avaliadores e avaliados; 
b) torna a acreditação das instituições e ciclos de estudos condição indispensável ao seu funcionamento e reconhecimento dependente de uma avaliação prévia favorável;

c) reconhece o papel fundamental das ordens e outras associações profissionais públicas, que passam a participar do processo de acreditação, fazendo cessar a sua anacrónica intervenção a posteriori no processo de reconhecimento profissional de cursos superiores tornada, em certos casos, necessária pela ausência de um sistema como o agora instituído.

\subsection{Expectativas Face ao Novo Modelo}

A necessidade assegurar que o sector do ensino superior contribui de forma adequada para a promoção do conhecimento em Portugal, conduziu a que se iniciasse um processo legislativo no sentido de garantir a qualidade do ensino superior, sobre o qual pairam as mais variadas expectativas.

O tratamento adequado de assuntos delicados como os presentes, exige que se parta de conceitos claros e de princípios esclarecedores acerca do sistema de avaliação, que sejam concordantes e harmoniosos com os objectivos e especificidades do ensino superior.

Por tal motivo, aguarda-se pela entrada em funcionamento da Agência de Avaliação e Acreditação do Ensino Superior, bem como das suas regras de funcionamento.

\section{CONCLUSÕES}

O processo de avaliação da qualidade das instituições de ensino superior esteve a cargo do CNAVES que, a partir de indicadores utilizados nas várias modalidades de ensino, produziu relatórios prospectivos e recomendações de racionalização e melhoria do sistema de ensino superior.

Em resultado das recomendações insertas no Relatório da ENQA de 2006, o processo de avaliação até então em vigor foi equacionado, tendo o Governo procedido à extinção do CNAVES e legislado no sentido de operacionalizar um novo sistema de avaliação e acreditação do ensino superior. $O$ actual regime jurídico para a garantia da qualidade do ensino superior em Portugal, acolhe muitas das ideias contidas nas recomendações da referida Associação.

Considera-se ser da maior importância a constituição de uma estrutura como a Agência de Avaliação e Acreditação do Ensino Superior, na prossecução de um ensino superior de qualidade e excelência, que possa concorrer com as melhores instituições europeias de ensino. Um ensino com estas características contribui para o desenvolvimento económico e progresso de Portugal.

A definição de critérios claros que visem a promoção do mérito e o progresso do conhecimento científico, favorece a competitividade das instituições de ensino superior, permitindo-lhes concorrer com as suas congéneres europeias.

\section{REFERENCIAS}

AZEVEDO, Feyo. A respeito do Relatório da ENQA Acreditação e Qualidade no Ensino Superior. Ingenium, II Série, nº 96, pp. 44-45, 2006.

BRICALL, J.M. Inform Universidad 2000. Conferência de Rectores de Universidades Españolas (CRUE), 2000. 
CARR, S.; HAMILTON, E.; MEADE, P. Is it possible? Investigating the influence of external quality audit on university performance. Quality in Higher Education, v. 11, n. 3, p. 195-211, Nov. 2005. doi:10.1080/13538320500329665

CONSELHO NACIONAL DE AVALIAÇÃO DO ENSINO SUPERIOR (CNAVES). Quality Assurance of Higher Education in Portugal. Disponível em: <http://www.cnaves.pt/>. Acesso em: jul. 2008.

EUROPEAN ASSOCIATION FOR QUALITY ASSURANCE IN HIGHER EDUCATION (ENQA). Disponível em: < http://www.enqa.eu/ > . Acesso em: set. 2008.

EUROPEAN ASSOCIATION FOR QUALITY ASSURANCE IN HIGHER EDUCATION (ENQA). Standards and Guidelines for Quality Assurance in the European Higher Education Area. 2005.

MORAN, M. The British regulatory state: high modernism and hyper-innovation. Oxford: University Press, 2007.

NATIONAL AGENCY FOR QUALITY ASSESSMENT AND ACCREDITATION OF SPAIN (ANECA). Encuentro sobre Instrumentos Comunes Metodológicos para la Evaluación y Acreditación en el Marco Europeo de la Declaración de Bolonia. Santander, Espanã, 2004.

NAUTA, P. et al. Accreditation models in higher education. ENQA - European Association for Quality Assurance in Higher Education, 2004.

ORGANIZAÇÃO PARA A COOPERAÇÃO E DESENVOLVIMENTO ECONÓMICO (OCDE). Reviews of National Policies for Education: Tertiary Education in Portugal, dez./2006.

ORSINGHER, C. Assessing Quality in European Higher Education Institutions: dissemination, methods and procedures. Heidelberg: Physica-Verlag, 2006.

PORTUGAL. Constituição de 1996.

PORTUGAL. Decreto-Lei no 205/98, de 11 de julho de 1998. Cria o Conselho Nacional de Avaliação do Ensino Superior (CNAVES).

PORTUGAL. Decreto-Lei $\mathbf{n}^{\mathbf{0}} \mathbf{7 4 / 2 0 0 6}$, de 24 de março 2006. Aprova o regime jurídico dos graus e diplomas, e se fixam os princípios gerais da acreditação das instituições de ensino superior e dos seus ciclos de estudos.

PORTUGAL. Decreto-Lei $\mathbf{n}^{\mathbf{0}}$ 369/2007, de 5 de novembro 2007. Criou a Agência de Avaliação e Acreditação do Ensino Superior.

PORTUGAL. Lei no 38/1994, de 21 de novembro 1994. Lei da Avaliação do Ensino Superior.

PORTUGAL. Lei n⿳0 38/2007, de 16 de agosto 2007. Lei da Avaliação do Ensino Superior.

PORTUGAL. Recomendação do Conselho n 98/561/CE, de 24 de setembro de 1998.

PORTUGAL. Ministério da Ciência, Tecnologia e Ensino Superior (MCTES). Regime jurídico de garantia da qualidade do ensino superior. Disponível em: 〈www.mctes.pt>. Acesso em: jul. 2008. 
PRØITZ T.; STENSAKER B.; HARVEY L. Accreditation, standards and diversity: an analysis of EQUIS accreditation reports. Assessment \& Evaluation in Higher Education, v. 29, n. 6, p. 735-750, Dec. 2004. doi:10.1080/0260293042000227263

SANTOS, S.M. Avaliação e acreditação. Disponível em: <http://jvcosta.planetaclix.pt/artigos/sms.html>. Acesso em: set. 2007.

SOGUEL, N.C; JACCARD, P. Governance and performance of education systems. Springer, 2007.

STENSAKER B. Trance, Transparency and transformation: the impact of external quality monitoring on higher education. Quality in Higher Education, v. 9, n. 2, p. 151-159, Aug. 2003. doi:10.1080/13538320308158

WESTERHEIJDEN, D.F; STENSAKER, B.; ROSA, M.J. Quality Assurance in Higher Education: trends in regulation, translation and transformation. Higher Education Dynamics, Springer, 2007.

WESTERHEIJDEN, D.F; STENSAKER, B.; ROSA, M.J. Quality as fashion: exploring the translation of a management idea into higher education. Netherlands: Springer, 2007. 